\title{
THE MYSTERIOUS NATURE OF HS $2331+3905$
}

\author{
$\therefore$ Araujo-B3nancor ${ }^{1,2}$ B. ' T. Gänsicke, ${ }^{3.2}$ H.-J. Hagen, ${ }^{4}$ T. R. Marsh, ${ }^{3,2}$ J. Thorstensen, ${ }^{5}$ E. T. Harlaftis, ${ }^{6}$ \\ R. E. Fried ${ }^{7}$ and D. Engels ${ }^{4}$
}

\begin{abstract}
RESUMEN
Informamos solme el descubrimiento de la única variable cataclísmica enana procedente del Hamburg Quasar Survey. HS 2:331 + 3905. Las of servaciones obtenidas durante tres años nos revelan un esquema inusual. La gran amplitud do $3.5 \mathrm{~h}$ de las variaciones 'n velocidad radial obtenidas a partir de espectroscopía óptica no es el períoko orbital del sistema. como se esperaría normalmente. Por el contrario, fotometría CCD extensiva, sugiere fucrtemente (1ue HS 2331+3905) es una variable cataclísmica de corto periodo orbital con un $P_{\text {orb }}=81.09$ min, ane (")nticne mula (nima blanci. la colal parece exhibir pulsaciones ZZ Ceti.
\end{abstract}

\section{ABSTRACT}

We report the discovery of one unique cataclysmic variable drawn from the Hamburg Quasar Survey, HS 23331+3905. Follow-up observations obtained over three years unveiled a very unusual picture. The large amplitude $3.5 \mathrm{~h}$ radial velocily variations obtained from our optical spectroscopy is not the orbital period of the system, as one wonld normally expect. Instead, extensive CCD photometry strongly suggests that HS 2:331+3905 is a short orbital period cataclysmic variable with $P_{\text {orb }}=81.09 \mathrm{~min}$, containing a cold white dwarf which appears to exhibit ZZ Ceti pulsations.

\section{key Words: BINARIES: CLOSE - NOVAE, CATACLYSMIC VARIABLES - STARS: INDIVIDUAL (HS 2331+3905)}

\section{INTRODUCIION}

HS $2331+3905$ (HS 2331 thereafter) was selected as a cal arelysmic variable (CV) candidate on the basis of its spectral characteristics in the Hamburg Quasar Survey (HQS; Hagen et al. 1995; Gänsicke et al. 200'2). The identification spectrum of HS 2331 contains broad double-peaked Balmer emission lines, (')al signs of the presence of an accretion disc, flanked by extremely broad absorptions throughs, indicating that this $\mathrm{CV}$ contains a relatively cold white dwall. The red part of the spectrum does not contain any spectral features that could be ascribed to the emission of the secondary. Here we report followup (ground and space) photometry and spectroscopy of HS 23:31. obtained over a three year period after its identification.

\section{OBSERVATIONS}

T'he left panel of Fig. 1 shows sample light curves of HS 2331 obtained from differential ('CD photometry. The morphology of the photonetric modulation

\footnotetext{
'Space Telescope Science Institute. ISS.

2 I niversity of Southampton, CK.

${ }^{3}$ Iniversity of Warwick. I'h.

${ }^{4}$ Hamburger Sternwarte. (iermany.

"Dartmouth College. CS:L

${ }^{6}$ Athens National ()bservatory. Greece.

${ }^{7}$ Braeside Obsevatory, Lish
}

is best described by a double-humped pattern with a period of $\sim 80 \mathrm{~min}$, with narrow dips centred on the observed minima between humps, which we identify as grazing eclipses. The higher time resolution data, in the middle and bottom panel of the same figure, reveal additional variability on time scales of $5 \mathrm{~min}$ and $1 \mathrm{~min}$. A period analysis of the entire CCD photometry of HS 2331 - more than 20000 data points - confirms the multiperiodic variability directly seen in the light curves. The likely orbital period derived from the period analysis, and ratified by folding all the data over it, is $P_{\text {orb }}=81.0852 \pm 0.0002 \mathrm{~min}$. In addition to the orbital period, the period analysis also reveals strong peaks at $83.38 \mathrm{~min}, 5.61 \mathrm{~min}$ and $1.12 \mathrm{~min}$. The $83.38 \mathrm{~min}$ is in the right frequency range to represent a superhump period (i.e. 1-2\% longer than $\left.P_{\text {orb }}\right)$. The power spectra around the $5.61 \mathrm{~min}$ and $1.12 \mathrm{~min}$ signals show an extremely complex structure indicative of a superposition of many frequencies. This type of structures are found in ZZCeti pulsators which can be explained by a number of non-radial pulsations modes, their harmonics, and various linear combinations of modes (o.g. Kotak et al. 2002).

Radial velocity variations of the Balmer and $\mathrm{He} I$ emission lines revealed yet another period at $\sim 3.5 \mathrm{~h}$. This period seems to drift throughout the three years 

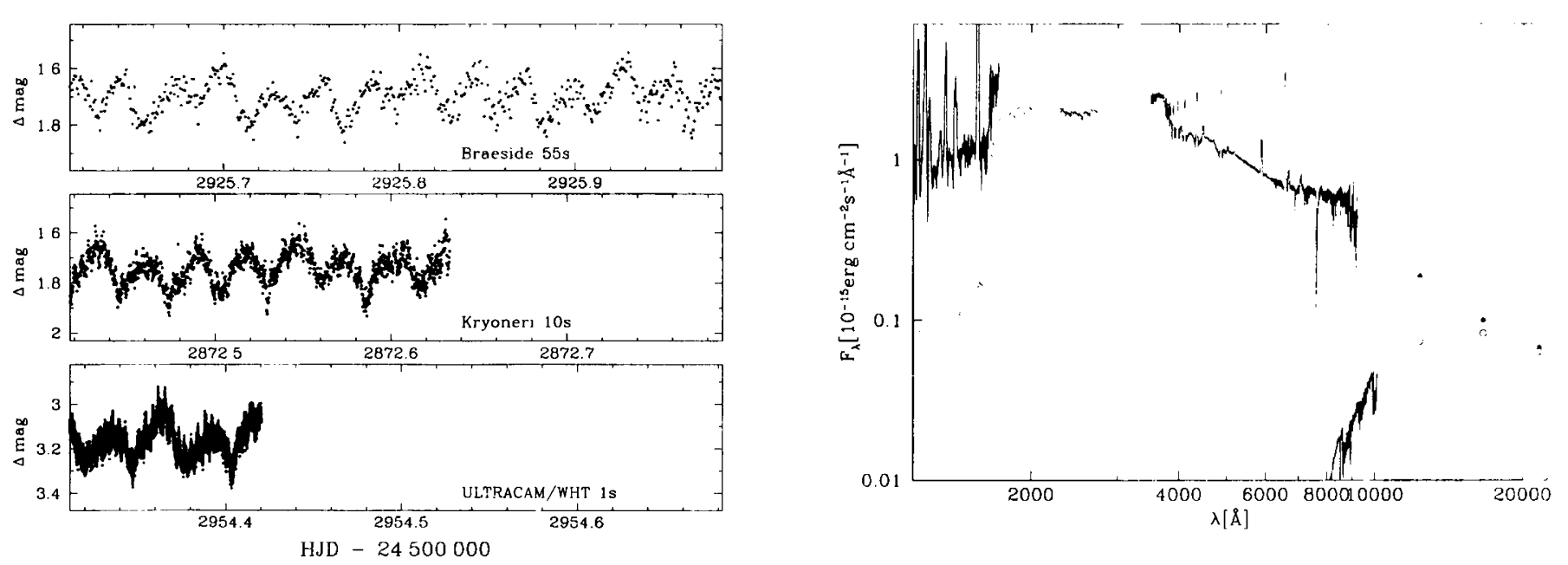

Fig. 1. Left panel: Samples of the light curves of HS 2331 obtained from differential C'C'D photometry. The names and numbers indicate the observatory and time resolution used in each of the obscrvations respectively. Right panel: Combination of FUV, optical spectra and 2MASS colours of HS2331 (dark line and filled circles) plotted with the best three-component model fit (grey line and open circles). See text for details.

of observations, and we could not identify a single period that will sacisfactorily fold all the available radial velocity data. We conclude that a persistent large-amplitude radial velocity variation with a period $\sim 3.5 \mathrm{~h}$ is present in HS 2331, however, this variation is not coherent but its period drifts on time scales of days.

In addition to optical spectroscopy we have obtained a HSTSTIS far-ultraviolet (FUV) spectrum of HS 2331. The $F U V$-optical spectra combined with the 2-MASS $J H K$ colours of HS 2331 allow us to confirm the presence of a low-temperature white dwarf of $T_{\mathrm{wd}} \sim 11000 \mathrm{~K}$, and to constrain the spectral type of the secondary to be later than $\mathrm{M} 9$, consistent with the short orbital period of the system (see right panel of Fig. 1). The accretion disc contribution to the spectral energy distribution of HS 2331 was matched with a $6500 \mathrm{~K}$ isothermal and isobaric slab and a surface density $1.81 \times 10^{-2} \mathrm{~g} \mathrm{~cm}^{-2}$. The distance of the system estimated from the white dwarf model fit is $\sim 100 \mathrm{pc}$, consistent with the large proper motion of the star, $\mu=0.14^{\prime \prime}$.

\section{DISCUSSION}

We have discovered a short orbital period system, HS 2331, as part of the HQS quest for new CVs. The orbital period of HS 2331, $P_{\text {orb }}=81.09 \mathrm{~min}$, was primarily defined by the detection of coherent eclipses. From our three years of photometric data, HS 2331 appears to be a permanent superhumper with $P_{\mathrm{SH}}=83.38 \mathrm{~min}$. The light curves of HS 2331 display double-humps with a period that is exactly half the orbital period (evident from a direct inspection of the light curves in the left panel of Fig. 1), suggesting that we are seeing some sort of symmetric structure, such as e.g. two bright spots. In addition, HS 2331, exhibits the photometric behaviour typical of ZZ Ceti pulsators, showing multifrequency variability in the range $\sim 60 \mathrm{~s}$ to $\sim 300 \mathrm{~s}$. The white dwarf temperature derived from our fit, $11000 \mathrm{~K}$, is well within the instability strip for ZZ Ceti pulsators. In order to disentangle the multiperiodic signature of the likely white dwarf pulsator in HS 2331, we need to organize a multi-site observing campaign to obtain long, continuous stretches of high time resolution photometry.

All in all, the pieces of the jigsaw seems to come together, and we are beginning to understand the nature of HS 2331. There are nerertheless, several points that we still need to address. The fact that the dominant radial velocity variability does not correspond to the orbital period of the system is particularly disconcerting. At present, we have no explanation for this phenomenon, nor for the physical significance of the $3.5 \mathrm{hr}$ radial velocity period. We are not aware of any other system suffering from this problem, but the reason for this may be that periods determined from radial velocity stidiles are usually adopted unquestioned as reflecting the corresponding orbital periods.

\section{REFERENCES}

Gänsicke, B. T., Hagen, H.-J., \& Engels, D. 200)2. ASP Conf. Ser., 261, 190

Hagen, H. J., Groote, D., Engels, D \& Reimers. D. 1995, A\&AS, 111, 195

hotak, R., van Kerkwijk, M.N. \& Clemens, J. C. '?(0)!, A\&A, $3 \times 8.219$ 